\title{
Prevalencia de triatominos infectados con Trypanosoma cruzi en el litoral de la ciudad de Arica
}

\author{
ALEJANDRO CARVAJAL*, JULIO ORELLANA*, WILSON WIGANT*, \\ CELIA BÓRQUEZ** y ISMELDALOBATO**

\section{PREVALENCE OF TRIATOMINES INFECTED WITH Trypanosoma cruzi IN THE COAST OF ARICA CITY}

It has been observed throughout America that when eradicating or diminishing the population of the domestic vector of Chagas'disease, the wild vectors adquires more epidemiological importance. For this reason the objective of this investigation was to determine if the wild vectors from the coast of Arica City are infected with Trypanosoma cruzi. The study was made in 52 triatomines captured from the coast of Arica city, during a period of 3 months since October until December 2004. Then, it was applied PCR method on these triatomines to find T. cruzi. The investigation showed that Mepraia gajardoi was infected with T. cruzi with a Trypano/triatomine index of 19.2\%, similar to the infection level found for Mepraia spinolai in other investigations.

Key words: Mepraia gajardoi, Trypanosoma cruzi, Polimerase Chain Reaction (PCR), Prevalence.

\section{INTRODUCCIÓN}

La enfermedad de Chagas es causada por un protozoo flagelado denominado Trypanosoma cruzi y existe en el continente americano hace cientos de años. Provoca grandes problemas económicos y de salud pública debido a que es una enfermedad crónica que causa discapacidad y muerte ${ }^{1,2}$. En Chile, el área endémica se distribuye entre las regiones primeras y sexta, existiendo alrededor de 150.000 personas infectadas y más de 850.000 personas con el riesgo de contraer la enfermedad ${ }^{3}$.

La transmisión del parásito al hombre se realiza principalmente por medio de los vectores biológicos (transmisión natural) ${ }^{4}$. En Chile, se describen 2 vectores de la enfermedad: el doméstico, Triatoma infestans, y el silvestre, Mepraia spinolai. Actualmente se ha descrito una tercera especie, también silvestre: Mepraia gajardoi, que habita las playas y desiertos costeros de la primera y segunda región ${ }^{3}$. La transmisión vectorial se lleva a cabo principalmente a través del vector del ciclo doméstico. Por este motivo, en Chile, se ha ejecutado el Programa Nacional de Control del Vector y de esta forma se está logrando la eliminación de $T$. infestans ${ }^{5,6}$.

Arica, que forma parte del área endémica del mal de Chagas en Chile, es una ciudad costera ubicada en la primera región de Tarapacá entre las coordenadas $18^{\circ} 28^{\prime}$ Latitud Sur y $70^{\circ} 19^{\prime}$ Longitud Oeste, extendiéndose unos $15 \mathrm{~km}$ al borde del Océano Pacífico ${ }^{7}$, con una población aproximada de 185.000 habitantes $^{8}$. Presenta un

\footnotetext{
* Tecnólogo Médico titulado de la Carrera de Tecnología Medica, Facultad de Ciencias de la Salud, Universidad de Tarapacá.

** Tecnólogo Médico Unidad de Parasitología, Facultad de Ciencias de la Salud, Universidad de Tarapacá.
} 
clima desértico costero, ideal para la subsistencia de los triatominos. De acuerdo a estudios realizados por el Departamento de Salud Ambiental, en los últimos 4 años, esta zona se encontraría libre de la transmisión vectorial de la enfermedad de Chagas. Sin embargo, durante el período de verano del año 2004, tras una prueba realizada en uno de estos insectos, hallado en el sector rocoso del litoral ariqueño, se comprobó que estaba infectado con T. cruzi, convirtiéndose en un peligro al constituir un posible foco de la infección para los humanos.

El objetivo de esta investigación fue determinar si los vectores silvestres del litoral de la ciudad de Arica se encuentran infectados con T. cruzi, demostrando la presencia del parásito en el contenido intestinal de los triatominos en estudio.

\section{MATERIAL Y MÉTODO}

El estudio se realizó sobre un total de 52 triatominos capturados, correspondientes a los estadios ninfales III, IV, V e imagos (macho y hembra). Se descartó la búsqueda de los triatominos de estadios I y II, en base a recopilación bibliográfica que reporta la baja o nula tasa de infección de estos estadios ${ }^{9-12}$. La captura se llevo a cabo en un periodo de 3 meses contemplados entre Octubre y Diciembre del año 2004. El lugar de captura seleccionado fue Guanera Culave, una zona costera situada aproximadamente a $13 \mathrm{~km}$ al sur de la ciudad de Arica. Este sector es de difícil acceso y es visitado esporádicamente por buzos, pescadores y algunos turistas.

Para la captura de los triatominos se utilizó la «captura activa». Ésta consiste en la búsqueda periódica de triatominos con el fin de capturarlos directamente. Para esta metodología se utilizó el «cebo vivo».

Una vez capturados, los triatominos fueron clasificados morfológicamente para determinar especie, estadio evolutivo y sexo ${ }^{4}$. Posteriormente, a los triatominos se le aplico la técnica de la Reacción en Cadena de la Polimerasa (PCR) para la búsqueda de T. cruzi, prueba confirmatoria en centros de referencia en la detección de la enfermedad de Chagas, debido a que distintos investigadores han confirmado que ésta es más sensible que el examen microscópico directo ${ }^{13}$. Para este análisis se utilizaron los primers $121 \mathrm{y}$ $122, \operatorname{los}$ que presentan un $100 \%$ de especificidad $^{14}$.

Los resultados obtenidos fueron analizados por medio del Método estadístico porcentual y la Prueba de Hipótesis para Diferencia de Proporciones ${ }^{15}$, con el fin de realizar comparaciones entre los diferentes sectores con respecto al índice de infección por T. cruzi y de acuerdo a los estadios evolutivos.

\section{RESULTADOS}

De un total de 52 triatominos se detectaron 7 $(17,1 \%)$ ninfas y $3(27,3 \%)$ imagos positivos para T. cruzi, con un índice Trypano/triatomino de $19,2 \%$, no existiendo una diferencia significativa en el nivel de infección entre los estadios ninfales e imagos, ni entre estadios ninfales. Estos resultados se presentan en la Tabla 1 y Figura 1.

\section{DISCUSIÓN}

De los 52 triatominos capturados y analizados morfológicamente, se determinó que el 100\% de ellos correspondían a M. gajardoi. Los datos obtenidos coinciden con los estudios realizados en $\mathrm{Chile}^{4}$, entre la primera región y región metropolitana, en el que se determinó que esta especie se encontraba ubicada en la costa de la primera y segunda región ${ }^{16}$. Otros estudios que corroboran estos resultados son los basados en citogenética, que sitúan a $M$. gajardoi en la misma zona geográfica ${ }^{17}$.

De un total de 52 triatominos analizados, 10 resultaron positivos a la infección por T. cruzi con un índice Trypano/triatomino de 19,2\%

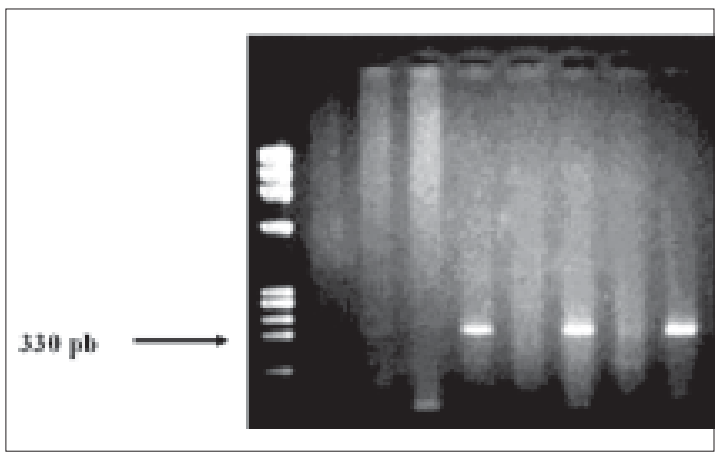

Figura 1. Electroforesis de ADN de Trypanosoma cruzi, observándose las bandas de 330 pb que indican la positividad de la muestra. 
Triatominos infectados con Trypanosoma cruzi en el litoral de la ciudad de Arica - A. Carvajal et al.

Tabla 1: Prevalencia de infección por Trypanosoma cruzi en triatominos del sector de Guanera Culave, según su estadio evolutivo. Arica, 2005

\begin{tabular}{|c|c|c|c|c|}
\hline Estadio & Número de muestra & Positivos & \multicolumn{2}{|c|}{ \%de infección } \\
\hline Ninfa III & 26 & 5 & $19,2 \%$ & $17,1 \%$ \\
\hline Ninfa IV & 8 & 1 & $12,5 \%$ & \\
\hline Nnfa V & 7 & 1 & $14,3 \%$ & \\
\hline Imago Macho & 5 & 3 & $60 \%$ & $27,3 \%$ \\
\hline Imago Hembra & 6 & 0 & $0 \%$ & \\
\hline Total & 52 & 10 & $19,2 \%$ & \\
\hline
\end{tabular}

(Tabla 1), resultado que no concuerda con lo observado por Frías et $\mathrm{al}^{4}$, quienes no encontraron ejemplares de $M$. gajardoi infectados con $T$. cruzi. Sin embargo, concuerda con el índice de infección observado en diversos estudios con M. spinolai, como en Combarbalá, IV región, donde se detectó que el $11,8 \%$ de $M$. spinolai estaba infectado con T. cruzi ${ }^{4}$. Resultados semejantes se obtuvieron con $M$. spinolai colectados en las áreas andinas de la III región hasta la Región Metropolitana, donde se detectaron tasas de infección por T. cruzi que variaron entre 11,5 a $25,8 \%{ }^{18}$. Con relación a otros triatominos, se ha reportado que en los Panstrongylus megistus recolectados de diferentes áreas de Brasil un nivel de infección cercano al $12 \%{ }^{19}$, valor similar al de este estudio.

Los resultados obtenidos en el sector revelaron que el $17,1 \%$ de las ninfas resultaron positivos para $T$. cruzi. No se detectó una diferencia significativa en el nivel de infección cuando se realizó la comparación entre estadios ninfales. Además el $27,3 \%$ de los imagos resultaron positivos para $T$. cruzi, no detectándose una diferencia significativa entre el nivel de infección de ninfas e imagos (Tabla 1). Esto concuerda con lo reportado en un estudio realizado con Triatoma sordida, en que se encontraron tanto ninfas como imagos infectados, presentando un índice de positividad para T. cruzi de $20,7 \%$ y $38,5 \%$, respectivamente $^{20}$. Canals et $\mathrm{al}^{21}$, también observaron que tanto las ninfas como los imagos de $M$. spinolai se encontraban infectados, con un promedio de infección por T. cruzi que varió entre un $4,8 \%$ en estadios ninfales, hasta un $58,33 \%$ en imagos.

El hallazgo de triatominos infectados por Tripanosoma cruzi podría explicarse por la existencia, en el sector de Guanera Culave, de animales que actuarían como reservorios de manteniendo el ciclo silvestre de la enfermedad de Chagas, tales como roedores y mamíferos marinos $^{22}$. Así, se ha observado que M. spinolai participa en el ciclo en el cual se encuentran Octodon degus (ratón cola de pincel) y Oryctolagus cuniculus (conejo), siendo estos las principales fuentes de alimento $^{23}$. También se debe considerar la poca accesibilidad que presenta Guanera Culave para las personas y que la tasa de infección en la población humana de la ciudad de Arica es baja, variando desde un $2,48 \%$ en el año 1986 hasta un 1,69\% en el año $2003^{24,25}$.

El nivel de infección encontrado en $M$. gajardoi muestra la importancia que presenta el seguir realizando diversos estudios en estos vectores, tales como la frecuencia de picada, preferencia por ciertos huéspedes, índice de alimentación con sangre humana, velocidad de deyección, etc, para establecer un perfil epidemiológico que permita determinar su rol en la dinámica de la transmisión de T. cruzi para los humanos.

\section{RESUMEN}

Se ha observado a lo largo de América que en la medida que se erradica o disminuye la población de los vectores domésticos de la enfermedad de Chagas, los vectores silvestres van cobrando mayor importancia epidemiológica. Por este motivo, el objetivo de esta investigación fue determinar si los vectores silvestres del litoral de la ciudad de Arica se encuentran infectados con Trypanosoma cruzi. El estudio se realizó en un total de 52 triatominos capturados en el litoral de la ciudad de Arica, entre Octubre y Diciembre del año 2004. utilizando la técnica de la Reacción en Cadena de la Polimerasa (PCR) para la búsqueda de T. cruzi. La investigación mostró 
que Mepraia gajardoi se encontraba infectado con T. cruzi presentando un índice Trypano/ triatomino de $19,2 \%$, similar a lo observado en Mepraia spinolai en otras investigaciones.

\section{REFERENCIAS}

1.- ATIAS A. Parasitología Médica. Editorial Mediterráneo. 4ª Edición, 2000.

2.- VARGAS D, WALLACE A, SOLARI A. Estandarización de la Reacción de la Polimerasa en Cadena (PCR) en muestras de sangre de ratones infectados con Trypanosoma cruzi. Parasitol Día 1996; 20: 136-40.

3.- OLEA A. Situación epidemiológica de la enfermedad de Chagas en Chile. Departamento de epidemiología, Ministerio de Salud, 2000.

4.- FRÍAS D. Mal de Chagas, huele a peligro. Revista Intramuros, n. 8, 2000.

5.- APT W. Enfermedad de Chagas ¿una zoonosis erradicable?. Rev Méd Chile 1994; 122: 1037-8.

6.- AGUILERA X, APT W, RODRÍGUEZ J, et al. Eficacia del control del vector de la enfermedad de Chagas demostrada a través de la infección humana. Rev Méd Chile 1994; 122: 259-64.

7.- ATLAS GEOGRÁFICO DE CHILE PARA LA EDUCACIÓN. Instituto Geográfico Militar. $3^{\mathrm{a}}$ edición, 1993.

8.- www.censo2002.cl

9.- BRENER Z, ANDRADE Z. Trypanosoma cruzi e Doença de Chagas. $2^{\mathrm{a}}$ edición. Editorial Guanabara Koogan, 2000.

10. SCHOFIELD C. Triatominae. Biología y control. Eurocommunica publications, West sussex, UK: 76, 1994.

11.- LINHARES A. Vetores do Trypanosoma cruzi. Revista de Patología Tropical 2000; 29 (supl): 83-8.

12.- CECERE M, CASTAÑEDA M, CANALE D, et al. Trypanosoma cruzi infection in a Triatoma infestans and other control program in rural northwestern Argentina. Rev Panan Salud Pública/Pan Am J Public Health, 1999.

13.- MUÑOZ C. Comparación de la Reacción de la Polimerasa en Cadena con la Detección Microscópica Directa de Trypanosoma cruzi en Triatoma infestans; Obtención del Índice Trypano/Triatomino. Universidad de Chile. Santiago, Chile, 2001.

14.- GARCÍA A. Diagnóstico de la infección trans- placentaria por Trypanosoma cruzi, por medio de la técnica de Reacción en Cadena de la Polimerasa, su aplicación en la evaluación del tratamiento médico y análisis génico-epidemiológico de las cepas infectantes. Universidad de Chile. Santiago, Chile. 54 p, 2000.

15.- DANIELS W. Bioestadística, base para el análisis de las ciencias de la salud. Editorial Limusa, S.A. México, 1999.

16.- FRÍAS D, HENRY A, GONZÁLEZ C. Mepraia gajardoi: a new species of Triatominae (Hemiptera: Reduviidae) from Chile and its comparison with Mepraia spinolai. Revista Chilena de Historia Natural 1998; 71: 177-88.

17.- PANZERA F, PÉREZ R, CALLEROS L, et al. Cytogenetic studies in Mepraia gajardoi (Heteroptera: Reduviidae). Chromosome behaviour in a spontaneous translocation mutant. European Journal of Entomology 2004; 101: 211-8

18.- SAGUA H, GONZÁlES J, ARAYA J, NEIRA I. Indicadores parasitológicos humanos de la presencia del niño en el norte de Chile. Unidad de parasitología. Facultad Ciencias de la Salud, Universidad de Antofagasta, 1999.

19.- SAGUA H, ARAYA J, GONZALES J, NEIRA I. $M$. spinolai in the southeastern pacific ocean coast (Chile) - First insular record and feeding pattern on the Pan de Azúcar Island. Mem. Inst. Oswaldo Cruz 2000; 95: 167-70.

20.- http://fai.unne.edu.ar/biologia/tesis/Bar/4.pdf

21.- CANALS M, EHRENFELD M, CATTAN P. Situación de Mepraia spinolai, vector silvestre de la enfermedad de Chagas en Chile, en relación con otros vectores desde la perspectiva de sus fuentes de alimentación. Rev Méd Chile 2000; 128: 10.

22.- RUIZ J. Propuesta de creación de santuario de la naturaleza «Cuevas de Anzota». Convenio CONAF e Ilustre Municipalidad de Arica, 1998.

23.- ACUÑA M. Efecto del hospedero sobre el crecimiento poblacional de Mepraia spinolai. Facultad de Medicina Veterinaria. Universidad de Chile, Chile. 2001. 67 p.

24.- PINTO H, MIQUELES M, MONTALVO M, et al. Investigación de Enfermedad de Chagas en habitantes de la I región. Chile. Facultad de Ciencias. Universidad de Tarapacá, 1986.

25.- Estadística de la Enfermedad de Chagas en donantes y no donantes del Hospital Dr. Juan Noé de Arica durante el período de 1992-2003. Laboratorio Banco de Sangre, Hospital Dr. Juan Noé, 2004. 